\title{
MINIMIZING POLYNOMIALS ON NONCOMPACT SETS
}

\author{
P.V. TrI, T.V.Q. SY
}

ABstraCt. In this paper, the problem of minimizing a polymonial $g_{*}=\inf _{x \in S(F)} g(x)$ in the noncompact case is investigated. It is known that such problem is severely ill-posed. This paper studies the representation of a non-negative polynomial $g$ on a noncompact semi-algebraic set $S$ modulo its KKT (Karush-Kuhn-Tucker) ideal. We use results of Demmel, Nie, Powers ([1]) and Nie, Demmel, Sturmfels ([6]) to solve it. A numerical example is given to illustrate the efficiency of the proposed method works.

2010 Mathematics Subject Classification: 30C45.

Keywords: Global optimization, polynomials, sum of squares, semidefinite programming.

\section{INTRODUCTION}

Given a polynomial function $g \in \mathbb{R}[x]=\mathbb{R}\left[x_{1}, x_{2}, \ldots, x_{n}\right]$ - the polynomial ring. Fix a finite subset $F=\left\{f_{1}, f_{2}, \ldots, f_{m}\right\} \subset \mathbb{R}[x]$. Denote

$$
S(F):=\left\{x \in \mathbb{R}^{n} \mid f_{i}(x) \geq 0, i=1, \ldots, m\right\}
$$

is the basic closed semialgebraic set generated by $F$. We consider the problem of minimizing a polymonial $g$ on $S(F): g_{*}=\inf _{x \in S(F)} g(x)$.

Finding the optimal solution of the problem $(*)$ is NP-hard problem (see [3], [5]). Based on the results of performing non-negative polynomials on the semi algebraic sets, some authors (eg, [2], [4], [8], ...) have developed a series of positive semidefinited programming ((SDP for short) (see [3], [5]) which their optimal values converge monotonically increasing to the optimum value of the problem $\left(^{*}\right)$. The idea traces back to work of Shor 1987 ([13]) and is further developed by Parrilo 2000 ([7]), by Lasserre 2001 ([2])and by Parrilo and Sturmfels 2003 ([8]).

We consider the general case: $S(F)=\mathbb{R}^{n}$. 
The purpose of this paper is to introdue the problem of minimizing a polymonial $g_{*}=\inf _{x \in S(F)} g(x)$ in the noncompact case. Uses results of positive performed theorems of Nie, Demmel, Sturmfels and Powers, we will build a series of positive semidefinited programming which their optimal values converge monotonically increasing to the optimum value $g_{*}$.

\section{PRELIMINARIES}

Given the polynomial ring $\mathbb{R}[x]=\mathbb{R}\left[x_{1}, x_{2}, \ldots, x_{n}\right]$.

Definition 1. $M \subset \mathbb{R}[x]$ is called the quadratic module if

$M+M \subset M, a^{2} M \subset M, \forall a \in \mathbb{R}[x]$ and $1 \in M$.

Definition 2. $P \subset \mathbb{R}[x]$ is called the preordering if

$P+P \subset P, P . P \subset P$ and $a^{2} \in P, \forall a \in \mathbb{R}[x]$.

Given a finite subset $F=\left\{f_{1}, f_{2}, \ldots, f_{m}\right\} \subset \mathbb{R}[x]$. Denote

$$
S(F):=\left\{x \in \mathbb{R}^{n} \mid f_{i}(x) \geq 0, i=1, \ldots, m\right\}
$$

is the basic closed semialgebraic set generated by $F$;

$$
M(F):=\left\{\sigma_{0}+\sigma_{1} f_{1}+\cdots+\sigma_{m} f_{m} \mid \sigma_{i} \in \sum \mathbb{R}[x]^{2}\right\}
$$

is the quadratic module in $\mathbb{R}[x]$;

$$
P(F):=\left\{\sum_{e \in\{0,1\}^{m}} \sigma_{e} f^{e} \mid \sigma_{e} \in \sum \mathbb{R}[x]^{2}, \forall e \in\{0,1\}^{m}\right\}
$$

is the preordering generated by $F$.

Definition 3. $M(F)$ is archimedean if $\exists k \geq 1 \mid k-\sum_{i=1}^{n} x_{i}^{2} \in M(F)$.

Example 1. Take $n=1, F=\left\{-x^{2}\right\} \subset \mathbb{R}[x]$. We have

$$
M(F)=\left\{\sigma_{0}-\sigma_{1} x^{2} \mid \sigma_{i} \in \sum \mathbb{R}[x]^{2}\right\}
$$

Take $k=1$. Then $k-x^{2}=1-x^{2} \in M(F)$. Thus $M(F)$ is archimedean. 
Example 2. Take $n=2, F=\left\{x-\frac{1}{2}, y-\frac{1}{2}, 1-x y\right\} \subset \mathbb{R}[x, y]$. Then

$$
M(F)=\left\{\sigma_{0}+\sigma_{1}\left(x-\frac{1}{2}\right)+\sigma_{2}\left(y-\frac{1}{2}\right)+\sigma_{3}(1-x y) \mid \sigma_{i} \in \sum \mathbb{R}[x, y]^{2}\right\} .
$$

We be alble to build quadratic module $Q \subset \mathbb{R}[x, y]$ ([5, Example 7.3.1]) which satisfies

$$
\left\{\begin{array}{l}
Q \cup-Q=\mathbb{R}[x, y], Q \cap-Q=\{0\}, \\
x-\frac{1}{2}, y-\frac{1}{2}, 1-x y \in Q,(\text { to } M(G) \subset Q), \\
k-\left(x^{2}+y^{2}\right) \notin Q, \forall k \in \mathbb{Z}, k \geq 1 .
\end{array}\right.
$$

Then $M(F) \subset Q, k-\left(x^{2}+y^{2}\right) \notin Q, \forall k \in \mathbb{Z}, k \geq 1$, and

$$
k-\left(x^{2}+y^{2}\right) \notin M(F), \forall k \in \mathbb{Z}, k \geq 1 .
$$

Thus $M(F)$ is not archimedean.

Theorem 1. ([10]) Suppose $S(F)$ is compact and $g \in \mathbb{R}[x]$. If $g>0$ on $S(F)$, then $g \in P(F)$.

Theorem 2. ([9]) Suppose $M(F)$ is archimedean and $g \in \mathbb{R}[x]$. If $g>0$ on $S(F)$, then $g \in M(F)$.

Remark 1. If $M(F)$ is archimedean, then $S(F)$ is compact.

The opposite of Remark 1 is not true. For example, we consider Example 2, we have

$$
S(F)=\left\{(x, y) \in \mathbb{R}^{2} \mid x-\frac{1}{2} \geq 0, y-\frac{1}{2} \geq 0,1-x y \geq 0\right\}
$$

is compact, and

$$
M(F)=\left\{\sigma_{0}+\sigma_{1}\left(x-\frac{1}{2}\right)+\sigma_{2}\left(y-\frac{1}{2}\right)+\sigma_{3}(1-x y) \mid \sigma_{i} \in \sum \mathbb{R}[x, y]^{2}\right\}
$$

is not archimedean.

\section{Semidefinited Programming (SDP)}

The problem SDP:

$$
\left\{\begin{array}{l}
\inf \sum_{i=1}^{n} c_{i} x_{i}, \\
G(x):=G_{0}+x_{1} G_{1}+\cdots+x_{n} G_{n} \succeq 0,
\end{array}\right.
$$

where $x=\left(x_{1}, \ldots, x_{n}\right) \in \mathbb{R}^{n}, c=\left(c_{1}, \ldots, c_{n}\right) \in \mathbb{R}^{n}$ and $G_{i} \in \operatorname{Sym}\left(\mathbb{R}^{d \times d}\right)$ is the symmetric matrix $(i=0, \ldots, n)$. 
Remark 2. Problem (1) can not achieve min. This can be seen in the following example.

Example 3. Consider the problem SDP

$$
\left\{\begin{array}{l}
p_{*}:=\inf x_{1} \\
\left(\begin{array}{cc}
x_{1} & 1 \\
1 & x_{2}
\end{array}\right) \succeq 0
\end{array}\right.
$$

We have $n=d=2, c^{T} x=\left(\begin{array}{ll}1 & 0\end{array}\right)\left(\begin{array}{l}x_{1} \\ x_{2}\end{array}\right)$ and

$$
F(x)=\left(\begin{array}{cc}
x_{1} & 1 \\
1 & x_{2}
\end{array}\right)=\underbrace{\left(\begin{array}{ll}
0 & 1 \\
1 & 0
\end{array}\right)}_{F_{0}}+x_{1} \underbrace{\left(\begin{array}{ll}
1 & 0 \\
0 & 0
\end{array}\right)}_{F_{1}}+x_{2} \underbrace{\left(\begin{array}{ll}
0 & 0 \\
0 & 1
\end{array}\right)}_{F_{2}} .
$$

Consider the equation $\operatorname{det}\left(\begin{array}{cc}x_{1}-\lambda & 1 \\ 1 & x_{2}-\lambda\end{array}\right)=0, \lambda \in \mathbb{R}$. Reduced, we obtain

$$
\lambda^{2}-\left(x_{1}+x_{2}\right) \lambda+x_{1} x_{2}-1=0 .
$$

The condition $G(x) \succeq 0$ is equivalent to eigenvalues of matric $G(x)$ is non negative. This is equivalent to Equation (2) has two non negative solutions, that is $S=\frac{-b}{a}=$ $x_{1}+x_{2} \geq 0$ and $P=\frac{c}{a}=x_{1} x_{2}-1 \geq 0$. Then $x_{1}>0, x_{2}>0$ and the objective function $c^{T} x=x_{1}$ can not achieve min on $\left\{x=\left(x_{1}, x_{2}\right) \in \mathbb{R}^{2} \mid x_{1}+x_{2} \geq 0, x_{1} x_{2}-1 \geq\right.$ $0\}$, and $p_{*}=0$.

The dual problem (DP for short) of (1) is

$$
\left\{\begin{array}{l}
\sup -\left\langle G_{0}, Z\right\rangle \\
\left\langle G_{i}, Z\right\rangle=c_{i}, i=1, \ldots, n \\
Z \succeq 0
\end{array}\right.
$$

\section{Remark 3.}

- SDP and DP are convex optimization problems. Using the polynomial algorithm to solve them.

- $O p t$ - value $(S D P) \geq O p t$ - value $(D P)$. 
4. The Case $M(F)$ is ARChimedean

For $g \in \mathbb{R}[x]$ and $S(F)$ is the basic closed semialgebraic set generated by $F$, we consider the problem

$$
g_{*}:=\inf \{g(x) \mid x \in S(F)\} .
$$

Remark 4. This is NP-hard problem. There is no efficient algorithm to solve it, unless the case $g$ is linear, $S(F)$ is convex polyhedron, then using the simplex algorithm to solve it.

Remark 5. For $\gamma \in \mathbb{R}$, test $g-\gamma \geq 0$ on $S(F)$ is generally difficult. However, test $g-\gamma \in M(F)$ can do (using SDP).

Corollary 3. $\sup _{g-\gamma \in M(F)} \gamma \leq g_{*}$.

Fix a positive integer $N \geq \operatorname{deg} g$. Denote

$$
\begin{gathered}
M_{N}(F):=\left\{\sum_{i=0}^{m} \sigma_{i} g_{i} \mid \sigma_{i} \in \sum \mathbb{R}[x]^{2}, \operatorname{deg}\left(\sigma_{i} g_{i}\right) \leq N, i=0, \ldots, m\right\}, \\
\chi_{N}:=\left\{L: \mathbb{R}[x]_{N} \rightarrow \mathbb{R} \text { linear } \mid L(1)=1, \text { and } L \geq 0 \text { on } M_{N}(F)\right\}, \\
g_{+, N}:=\inf \left\{L(g) \mid L \in \chi_{N}\right\}, \\
g_{N}^{*}:=\sup \left\{\gamma \in \mathbb{R} \mid g-\gamma \in M_{N}(F)\right\} .
\end{gathered}
$$

Proposition 1. ([4])

(a) $g_{N}^{*} \leq g_{+, N} \leq g_{*}$.

(b) $g_{+, N} \leq g_{+, N+1} ; g_{N}^{*} \leq g_{N+1}^{*}$.

(c) If $M(F)$ is archimedean, then $\lim _{N \rightarrow \infty} g_{N}^{*}=g_{*}$. Hence $\lim _{N \rightarrow \infty} g_{+, N}=g_{*}$.

Proposition 2. Problem (4) is SDP.

Proof. Without loss generality, we assume $f_{i} \not \equiv 0$ and $\operatorname{deg} f_{i} \leq N, i=1, \ldots, m$. Because if $\operatorname{deg}\left(\sigma_{i} f_{i}\right) \leq N$ and $\operatorname{deg} f_{i}>N$, then $\sigma_{i}=0$, so $\sigma_{i} f_{i}=0$ : not have any contribution to $M_{N}(F)$. We see $\mathbb{R}[x]_{N}$ generated by the basic set $\left\{x^{\alpha}|| \alpha \mid \leq N\right\}$, number of elements of that basic is $C_{n+N}^{N}$. We consider linear mapping

$$
L: \mathbb{R}[x]_{N} \longrightarrow \mathbb{R}, L(p)=L\left(\sum_{|\alpha| \leq N} p_{\alpha} x^{\alpha}\right)=\sum_{|\alpha| \leq N} p_{\alpha} L\left(x^{\alpha}\right) .
$$


Putting $y_{\alpha}=L\left(x^{\alpha}\right),|\alpha| \leq N$ then $L$ corresponds to a vector $\left(y_{\alpha}\right),|\alpha| \leq N, y_{\alpha} \in \mathbb{R}$. We have $y_{0}=1$. $L \geq 0$ on $M_{N}(F)$ is equivalent to

$$
L\left(\sum_{i=0}^{m} \sigma_{i} f_{i}\right) \geq 0, \sigma_{i} \in \sum \mathbb{R}[x]^{2}, \operatorname{deg}\left(\sigma_{i} f_{i}\right) \leq N,
$$

or

$$
\sum_{i=0}^{m} L\left(\sigma_{i} f_{i}\right) \geq 0, \sigma_{i} \in \sum \mathbb{R}[x]^{2}, \operatorname{deg}\left(\sigma_{i} f_{i}\right) \leq N
$$

or

$$
L\left(\sigma_{i} f_{i}\right) \geq 0, \forall i, \sigma_{i} \in \sum \mathbb{R}[x]^{2}, \operatorname{deg}\left(\sigma_{i} f_{i}\right) \leq N
$$

or

$$
L\left(p^{2} f_{i}\right) \geq 0, p \in \mathbb{R}[x], \operatorname{deg} p \leq \frac{N-\operatorname{deg}\left(f_{i}\right)}{2} .
$$

Test

$$
\operatorname{deg} p \leq \frac{N-\operatorname{deg} f_{i}}{2} .
$$

Indeed, since $p^{2} f_{i} \in M_{N}(F)$ we have

$$
\operatorname{deg}\left(p^{2} f_{i}\right) \leq N
$$

or

$$
\operatorname{deg} p^{2}+\operatorname{deg} f_{i} \leq N
$$

or

$$
2 \operatorname{deg} p+\operatorname{deg} f_{i} \leq N
$$

or

$$
\operatorname{deg} p \leq \frac{N-\operatorname{deg} f_{i}}{2} .
$$

We write $g=\sum_{|\alpha| \leq N} g_{\alpha} x^{\alpha}$, thus

$$
L(g)=\sum_{|\alpha| \leq N} g_{\alpha} L\left(x^{\alpha}\right)=\sum_{|\alpha| \leq N} g_{\alpha} y_{\alpha}=g_{0}+\sum_{|\alpha| \leq N, \alpha \neq 0} g_{\alpha} y_{\alpha} .
$$

If $p=\sum_{\alpha} p_{\alpha} x^{\alpha}$, then $p^{2}=\sum_{\alpha, \beta} p_{\alpha} p_{\beta} x^{\alpha+\beta}$, therefore

$$
L\left(p^{2}\right)=\sum_{\alpha, \beta} p_{\alpha} p_{\beta} L\left(x^{\alpha+\beta}\right)=\sum_{\alpha, \beta} p_{\alpha} p_{\beta} y_{\alpha+\beta} .
$$


We write $f_{i}=\sum_{\gamma} f_{i \gamma} x^{\gamma}$. Similar to the above, we have

$$
\begin{gathered}
p^{2} f_{i}=\sum_{\alpha, \beta} p_{\alpha} p_{\beta} x^{\alpha+\beta} f_{i}=\sum_{\alpha, \beta, \gamma} p_{\alpha} p_{\beta} f_{i \gamma} x^{\alpha+\beta+\gamma} \\
\mathrm{v} L\left(p^{2} f_{i}\right)=\sum_{\alpha, \beta, \gamma} p_{\alpha} p_{\beta} f_{i \gamma} y_{\alpha+\beta+\gamma}=\sum_{\alpha, \beta}\left(\sum_{\gamma} f_{i \gamma} y_{\alpha+\beta+\gamma}\right) p_{\alpha} p_{\beta} . \text { Putting } \\
M\left(f_{i} * y\right)=\left(\sum_{\gamma} f_{i \gamma} y_{\alpha+\beta+\gamma}\right)_{\alpha, \beta} .
\end{gathered}
$$

Then, $M\left(f_{i} * y\right)$ is the matrix which size is $D_{i} \times D_{i}$, where

$$
D_{i}=\#\left\{\alpha|| \alpha \mid \leq \frac{N-\operatorname{deg} f_{i}}{2}\right\} .
$$

Note that $M(1 * y)=M(y)$. Then

$$
L\left(p^{2} f_{i}\right)=\sum_{\alpha, \beta}\left(\sum_{\gamma} f_{i \gamma} y_{\alpha+\beta+\gamma}\right) p_{\alpha} p_{\beta}=p^{T} M\left(f_{i} * y\right) p .
$$

Therefore, condition $L\left(p^{2} f_{i}\right) \geq 0$ is equivalent to $p^{T} M\left(f_{i} * y\right) p \geq 0$. This is equivalent to $M\left(f_{i} * y\right) \succeq 0$. Thus

$$
L \in \chi_{N} \Leftrightarrow\left\{\begin{array} { l } 
{ L ( 1 ) = 1 , } \\
{ L \geq 0 \text { on } M _ { N } ( G ) }
\end{array} \Leftrightarrow \left\{\begin{array}{l}
y_{0}=1, \\
M\left(f_{i} * y\right) \succeq 0, i=0, \ldots, m .
\end{array}\right.\right.
$$

Putting $G(y):=\operatorname{diag}\left(M\left(f_{i} * y\right), \ldots, M\left(f_{i} * y\right)\right)$. The size of the matrix $G(y)$ is $\sum_{1=0}^{m} D_{i} \times \sum_{1=0}^{m} D_{i}$. Then,

$$
\left\{\begin{array} { l } 
{ y _ { 0 } = 1 } \\
{ M ( f _ { i } * y ) \succeq 0 , i = 0 , \ldots , m }
\end{array} \Leftrightarrow \left\{\begin{array}{l}
y_{0}=1 \\
G(y) \succeq 0
\end{array}\right.\right.
$$

For $|\alpha| \leq N$, we define $e^{(\alpha)}:=\left(e_{\beta}^{(\alpha)}\right)$, where

$$
e_{\beta}^{(\alpha)}:= \begin{cases}0, & \text { if } \beta \neq \alpha \\ 1, & \text { if } \beta=\alpha\end{cases}
$$


So $\left\{e^{(\alpha)}, \alpha \neq 0\right\}$ is basic vector of freedom variables space $y=\left(y_{\alpha}\right),|\alpha| \leq N, \alpha \neq 0$, that is $y=\sum y_{\alpha} e^{(\alpha)}, \forall y=\left(y_{\alpha}\right),|\alpha| \leq N, \alpha \neq 0$. Then $G(y)=G_{0}+\sum_{|\alpha| \leq N, \alpha \neq 0} y_{\alpha} G^{\alpha}$, $G_{\alpha}:=G\left(e^{(\alpha)}\right)$, and

$$
\left\{\begin{array} { l } 
{ y _ { 0 } = 1 , } \\
{ G ( y ) \succeq 0 }
\end{array} \Leftrightarrow \left\{\begin{array}{l}
y_{0}=1, \\
G_{0}+\sum_{|\alpha| \leq N, \alpha \neq 0} y_{\alpha} G^{\alpha} \succeq 0 .
\end{array}\right.\right.
$$

So $g_{+, N}:=\inf \left\{L(g) \mid L \in \chi_{N}\right\}=\inf \left\{g_{0}+\sum_{\alpha \neq 0} g_{\alpha} y_{\alpha}\right\}=g_{0}+\inf \sum_{\alpha \neq 0} g_{\alpha} y_{\alpha}$. We see that problem calculate $g_{+, N}$ with constrain $L \in \chi_{N}$ same as problem calculate $g_{0}+\inf \sum_{\alpha \neq 0} g_{\alpha} y_{\alpha}$ with constrain

$$
\left\{\begin{array}{l}
y_{0}=1, \\
G_{0}+\sum_{|\alpha| \leq N, \alpha \neq 0} y_{\alpha} G^{\alpha} \succeq 0,
\end{array}\right.
$$

or with constrain $G(y) \succeq 0$. Therefore Problem (4) is SDP.

Proposition 3. Problem (5) is duality of Problem (4).

Proof. Take $\gamma \in \mathbb{R}$ so that $g-\gamma=\sigma_{0}+\sigma_{1} f_{1}+\cdots+\sigma_{m} f_{m}$, where

$$
\sigma_{i} \in \sum \mathbb{R}[x]^{2}, \operatorname{deg} \sigma_{i} \leq \frac{N-\operatorname{deg} f_{i}}{2}, i=0, \ldots, m .
$$

For $\sigma_{i} \in \sum \mathbb{R}[x]^{2}$, there exists a positive semidefinite (PSD for short) matrix which size is $D_{i} \times D_{i}: A^{(i)}=\left(A_{\delta \beta}^{(i)}\right)_{\delta, \beta}$ so that $\sigma_{i}=\sum_{\delta, \beta} A_{\delta \beta}^{(i)} x^{\delta+\beta}$. Then

$$
g-\gamma=\sum_{i=0}^{m} \sigma_{i} f_{i}=\sum_{i=0}^{m} \sum_{\delta, \beta} A_{\delta \beta}^{(i)} x^{\delta+\beta} f_{i} .
$$

We write $f_{i}=\sum_{\gamma} f_{i \gamma} x^{\gamma}$. Then

$$
g-\gamma=\sum_{i=0}^{m} \sum_{\delta, \beta} \sum_{\gamma} A_{\delta \beta}^{(i)} f_{i \gamma} x^{\delta+\beta+\gamma} .
$$

For

$$
g=\sum_{\alpha} g_{\alpha} x^{\alpha}=g_{0}+\sum_{\alpha \neq 0} g_{\alpha} x^{\alpha}
$$


we have

$$
g_{0}+\sum_{\alpha \neq 0} g_{\alpha} x^{\alpha}-\gamma=\sum_{i=0}^{m} \sum_{\delta, \beta} \sum_{\gamma} A_{\delta \beta}^{(i)} f_{i \gamma} x^{\delta+\beta+\gamma}
$$

or

$$
g_{0}-\gamma+\sum_{\alpha \neq 0} f_{\alpha} x^{\alpha}=\sum_{i=0}^{m} \sum_{\delta, \beta} \sum_{\gamma} A_{\delta \beta}^{(i)} f_{i \gamma} x^{\delta+\beta+\gamma} .
$$

Identify coefficients two sides the above equation, we get

$$
\left\{\begin{array}{l}
g_{0}-\gamma=\sum_{i=0}^{m} A_{00}^{(i)} f_{i 0}=\left\langle G_{0}, A\right\rangle, \\
g_{\alpha}=\sum_{i=0}^{m} \sum_{\delta+\beta+\gamma=\alpha} A_{\delta \beta}^{(i)} f_{i \gamma}=\left\langle G_{\alpha}, A\right\rangle, \text { for } \alpha \neq 0,
\end{array}\right.
$$

where $A:=\operatorname{diag}\left(A^{(0)}, \ldots, A^{(m)}\right), G_{\alpha}:=G\left(e^{(\alpha)}\right)$. We have $A$ is PSD and

$$
\begin{aligned}
g_{N}^{*} & =\sup \left\{\gamma \mid g-\gamma \in M_{N}(F)\right\} \\
& =\sup \left\{g_{0}-\left\langle G_{0}, A\right\rangle \mid A \succeq 0, g_{\alpha}=\left\langle G_{\alpha}, A\right\rangle, \alpha \neq 0\right\} \\
& =g_{0}+\sup \left\{-\left\langle G_{0}, A\right\rangle \mid A \succeq 0, g_{\alpha}=\left\langle G_{\alpha}, A\right\rangle, \alpha \neq 0\right\} .
\end{aligned}
$$

Thus, Problem (5) is duality of Problem (4).

Remark 6. Can happen case $g_{N}^{*} \neq g_{+, N}$. However, if $M(F) \cap-M(F)=\{0\}$, then $g_{N}^{*}=g_{+, N}$. (See [5, Proporition 10.5.1]).

Example 4. [3, Problem 4.6, 4.7] We consider the optimization problem

$$
\left\{\begin{array}{l}
\inf _{x} g(x):=-x_{1}-x_{2} \\
x_{2} \leq 2 x_{1}^{4}-8 x_{1}^{3}+8 x_{1}^{2}+2 \\
x_{2} \leq 4 x_{1}^{4}-32 x_{1}^{3}+88 x_{1}^{2}-96 x_{1}+36 \\
0 \leq x_{1} \leq 3,0 \leq x_{2} \leq 4
\end{array}\right.
$$

Then $g_{4}^{*}=g_{*}=-5.5079$.

Example 5. [3, Problem 4.6, 4.7] We consider the optimization problem

$$
\left\{\begin{array}{l}
\inf _{x} g(x):=-12 x_{1}-7 x_{2}+x_{2}^{2} \\
-2 x_{1}^{4}+2-x_{2}=0 \\
0 \leq x_{1} \leq 2,0 \leq x_{2} \leq 3
\end{array}\right.
$$

Then $g_{5}^{*}=g_{*}=-16.73889$. 


\section{The Case $M(F)$ is not archimedean}

We have the same results as above if we replace the quadratic module $M_{N}(F)$ by the preordering

$$
P_{N}(F):=\left\{\sum_{e \in\{0,1\}^{m}} \sigma_{e} f^{e} \mid \sigma_{e} \in \sum \mathbb{R}[x]^{2}, \operatorname{deg} \sigma_{e} f^{e} \leq N, e \in\{0,1\}^{m}\right\} .
$$

We denote

$$
\begin{gathered}
\chi_{N}:=\left\{L: \mathbb{R}[x]_{N} \rightarrow \mathbb{R} \text { linear } \mid L(1)=1 \text { and } L \geq 0 \text { on } P_{N}(F)\right\}, \\
g_{+, N}:=\inf \left\{L(g) \mid L \in \chi_{N}\right\}, \\
g_{N}^{*}:=\sup \left\{\gamma \in \mathbb{R} \mid g-\gamma \in P_{N}(F)\right\} .
\end{gathered}
$$

\section{Proposition 4.}

(a) $g_{N}^{*} \leq g_{+, N} \leq g_{*}$.

(b) $g_{+, N} \leq g_{+, N+1} ; g_{N}^{*} \leq g_{N+1}^{*}$.

(c) If $S(F)$ is compact, then $\lim _{N \rightarrow \infty} g_{N}^{*}=g_{*}$. Hence $\lim _{N \rightarrow \infty} g_{+, N}=g_{*}$.

Proof. (a)We prove $g_{+, N} \leq g_{*}$. Taking arbitrary $a \in S(F)$, define

$$
L_{a}: \mathbb{R}[x]_{N} \rightarrow \mathbb{R}, L_{a}(q)=q(a) .
$$

We have $L_{a}(1)=1, L_{a}\left(\sum_{e \in\{0,1\}^{m}} \sigma_{e} f^{e}\right)=\sum_{e \in\{0,1\}^{m}} L_{a}\left(\sigma_{e} f^{e}\right)=\sum_{e \in\{0,1\}^{m}} \sigma_{e} f_{e}(a) \geq 0$.

Then $L_{a} \in \chi_{N}$. Because

$$
g_{+, N}:=\inf \left\{L(g) \mid L \in \chi_{N}\right\}
$$

we get

$$
g_{+, N} \leq L_{a}(g)=g(a) .
$$

By $a \in S(F)$ is arbitrary, we have

$$
g_{+, N} \leq \inf _{a \in S(F)} g(a)=g_{*}
$$

Next, we prove $g_{N}^{*} \leq g_{+, N}$. Take $\gamma \in \mathbb{R}$ such that $g-\gamma \in P_{N}(F)$ and $L \in \chi_{N}$ is arbitrary. We have

$$
0 \leq L(g-\gamma)=L(g)-L(\gamma)=L(g)-\gamma
$$


Then $L(g \geq \gamma$. Therefore

$$
\inf \left\{L(g) \mid L \in \chi_{N}\right\} \geq \sup \left\{\gamma \in \mathbb{R} \mid g-\gamma \in P_{N}(F)\right\},
$$

that is $g_{+, N} \geq g_{N}^{*}$.

(b) We have $P_{N}(F) \subseteq P_{N+1}(F)$ and $\chi_{N+1} \subseteq \chi_{N}$. Take $\gamma \in \mathbb{R}$ such that

$$
g-\gamma \in P_{N}(F)
$$

we get $g-\gamma \in P_{N+1}(F)$. Thus $g_{N}^{*} \leq g_{N+1}^{*}$.

Next, we prove $g_{+, N} \leq g_{+, N+1}$. Take $L \in \chi_{N+1}$ is abitrary. Put

$$
L^{\prime}:=L_{\mid \mathbb{R}[x]_{N}},
$$

then $L^{\prime} \in \chi_{N}$ and $L^{\prime}(g)=L(g)$. Therefore

$$
\inf \left\{L(g) \mid L \in \chi_{N}\right\} \leq \inf \left\{L(g) \mid L \in \chi_{N+1}\right\},
$$

that is $g_{+, N} \leq g_{+, N+1}$.

(c) Take $\gamma \in \mathbb{R}, \gamma<g_{*}$. We have $g-\gamma>0$ on $S(G)$. From Theorem 1 , we get

$$
g-\gamma \in P(F), \text { that is } g-\gamma=\sum_{e \in\{0,1\}^{m}} \sigma_{e} f^{e}
$$

where $\sigma_{e} \in \sum \mathbb{R}[x]^{2}$. Choose $N=\max \operatorname{deg}\left(\sigma_{e} f^{e}\right)$, then $g-\gamma \in P_{N}(F)$, so $\gamma \leq g_{N}^{*}$. Thus

$$
\gamma \leq g_{N}^{*} \leq g_{*}
$$

For $\gamma \uparrow g_{*}$, then $g_{N}^{*} \uparrow g_{*}$. From $g_{N}^{*} \stackrel{N \rightarrow \infty}{\longrightarrow} g_{*}$ and $g_{N}^{*} \leq g_{+, N} \leq g_{*}$, we obtain $g_{+, N} \stackrel{N \rightarrow \infty}{\longrightarrow} g_{*}$.

Proposition 5. Problem (6) is SDP.

Proof. Similar to the proof of Proposition 2.

Proposition 6. Problem (7) is duality of Problem (6).

Proof. Similar to the proof of Proposition 3. 
Example 6. We consider problem

$$
\left\{\begin{array}{l}
\inf _{(x, y) \in S}(x, y)=x+y \\
S=\left\{(x, y) \in \mathbb{R}^{2} \mid x \geq \frac{1}{2}, y \geq \frac{1}{2}, x y \leq 1\right\}
\end{array}\right.
$$

Then

$$
g_{2}^{*}=g_{*}=1
$$

Example 7. Problem

$$
\left\{\begin{array}{l}
\inf _{(x, y) \in S} g(x, y)=-x-y \\
S=\left\{(x, y) \in \mathbb{R}^{2} \mid x \geq \frac{1}{2}, y \geq \frac{1}{2}, x y \leq 1\right\}
\end{array}\right.
$$

has

$$
g_{2}^{*}=g_{*}=-2,5
$$

\section{Conclusion}

The paper found out the problem of minimizing a polymonial $g_{*}=\inf _{x \in S(F)} g(x)$ in case $S(F)$ is compact, where $g \in \mathbb{R}[x]$ and $S(F)$ is the basic closed semialgebraic set generated by $F$.

The paper presented positive performed theorems:

- Putinar,

- Schmüdgen.

Using results of positive performed theorems of Putinar ([9]) and Schmüdgen ([10]), we can build a series of positive semidefinited programming which their optimal values converge monotonically increasing to the optimum value $g_{*}$. Finally, the numerical results show that the proposed method works effectively.

\section{REFERENCES}

[1] J. Demmel, J. Nie, V. Powers, Representations of positive polynomials on noncompact simialgebraic sets via KKT ideals, Journal of Pure and Applied algebra, 207 (2007), 189-200.

[2] J. B. Lassere, Global optimization with polynomials and the problem of moments, SIAM J. Optim., 11, No. 3 (2001), 796-817. MR1814045. Zbl 1010.90061. 
P.V. Tri, T.V.Q. Sy - Minimizing polynomials on noncompact sets

[3] M. Laurent, Sums of square, moment matrices and optimization over polynomials, Emerging applications of algebraic geometry, 157-270, IMA vol. Math.Appl, 149, Springer, Newyork, 2009. MR2500468.

[4] M. Marshall, Optimization of polynomial functions, Canadian Math. Bull., 46 (2003), 575-587. MR2011395. Zbl 1063.14071.

[5] M. Marshall, Positive polynomials and sums of squares, Mathematical Surveys and Monographs, AMS, 146, 2008. MR2383959.

[6] J. Nie, J. Demmel, and B. Sturmfels, Minimizing polynomials via sums of squares over the gradient ideal, Mathematical Programming Series A, 106, (2006), 587-606. MR2216797.

[7] P.A. Parrilo, Structured semidefinite programs and semialgebraic geometry methods in robustness and optimization, Ph.D. thesis, California Institute of Technology, May 2000.

[8] P.A. Parrilo, and B. Sturmfels, Minimizing polynomial functions, DIMACS Series in Discrete Mathematices and Theoretical Computer Science, AMS, 60 (2003), 83-99. Zbl 1099.13516.

[9] M. Putinar, Positive polynomials on compact semialgebraic sets, Ind. Univ. Math. J. 43, No 3 (1993), 969-984.

[10] K. Schmüdgen, The K-moment problem for compact semialgebraic sets, Math. Ann. 289, No. 2 (1991), 203-206.

[11] M. Schweighofer, Optimization of polynomials on compact semialgebraic sets, SIAM J. Optim., 15 (3) (2005), 805-825. MR2142861. Zbl 1114.90098

[12] M. Schweighofer, Global optimization of polynomials using gradient tentacles and sums of squares, SIAM J. Optim., 17 (3) (2006), 920-942. Zbl 1118.13026.

[13] N.Z. Shor, An approach to obtaining global extremums in polynomial mathematical programming problems, Kibernetika, 5 (1987), 102-116.

[14] H.H. Vui, P.T. Son, Global optimization of polynomials using the truncated tangency variety and sums of squares, SIAM J. Optim, 19 (2) (2008), 941-951. Zbl 1163.13020 .

[15] H.H. Vui, P.T. Son, Representations of positive polynomials and optimization on noncompact semialgebraic sets, SIAM J. Optim, 20 (6) (2010), 3082-3130. Zbl 1279.14071 .

P. V. Tri

Faculty of Foundation Sciences, Telecommunications University, 
Khanh Hoa province, Viet Nam

email: phanvantri82@gmail.com

T. V. Q. Sy

Military Information Technology Institute,

Academy of Military Science and Technology,

Ho Chi Minh City, Viet Nam

email:sytvq@yahoo.com 